\title{
Medial Tibial Defect Reconstruction in Primary Total Knee Arthroplasty Using Sculco'sTechnique
}

\author{
Jacob Eapen, N. Selvam, Lingaraj, Syed.N \\ Department of Orthopaedics, Pondicherry Institute Of Medical Sciences,Puducherry
}

\begin{abstract}
Total knee Repalcement is a wellaccepted and routine surgeryperformed for patients with severe osteoarthritis of knee who doesnot show any improvement with analgesics,physiotherapy and intra-articular steroid injections and visco-supplements. Though surgical techniques of Total Knee Replacement have evolved over the years, total knee arthroplasty in a patient with medial tibial defect remains challenging.

We came across a patient with severe varusknee with medial tibial defect.

He underwent medial tibial reconstruction and Total Knee Replacement. Post operatively patient attained full range of movements and started full weight bearing at the end of 2 months. We recommend that Sculco's Technique of reconstruction is appropriate treatment for these type of cases.
\end{abstract}

\section{Introduction:}

In Primary total knee arthroplasty the defects are usually secondary to severe malalignment and are associated with destructive changes in articular cartilage,bone and surrounding soft tissues.Various classifications have been described for medial tibialdefect.Anderson orthopaedic research institute ${ }^{1}$ classifies the defect into three types. Type 1- minor defect that does not compromise the stability.Type 2: Loss of cancellous bone requiring reconstruction, A: one condyle,B: both condyles.Type 3: Bone loss that compromise the major portion of the condyle.

The treatment of the tibialdefect in primary total knee arthroplasty depends on whether it is a contained or uncontained defect and the size of the defect.Based on the size it is divided into small $(<5 \mathrm{~mm})$ and $\operatorname{large}(>5 \mathrm{~mm})$. defect.

It is essential to have good quality Xray for proper preoperativeplanning as well as to classify the

The various methods to treat tibial defects are; translation of the component away from thedefect,lowertibialresection,polymethylmethacrylatecement filling $+/$ - screws, autografts ,metallic wedge augments or using custom implants.

This study describes the treatment and outcome of using autograft for reconstructing large medial tibial defects ( $>5 \mathrm{~mm}$ ) using Sculco's technique.

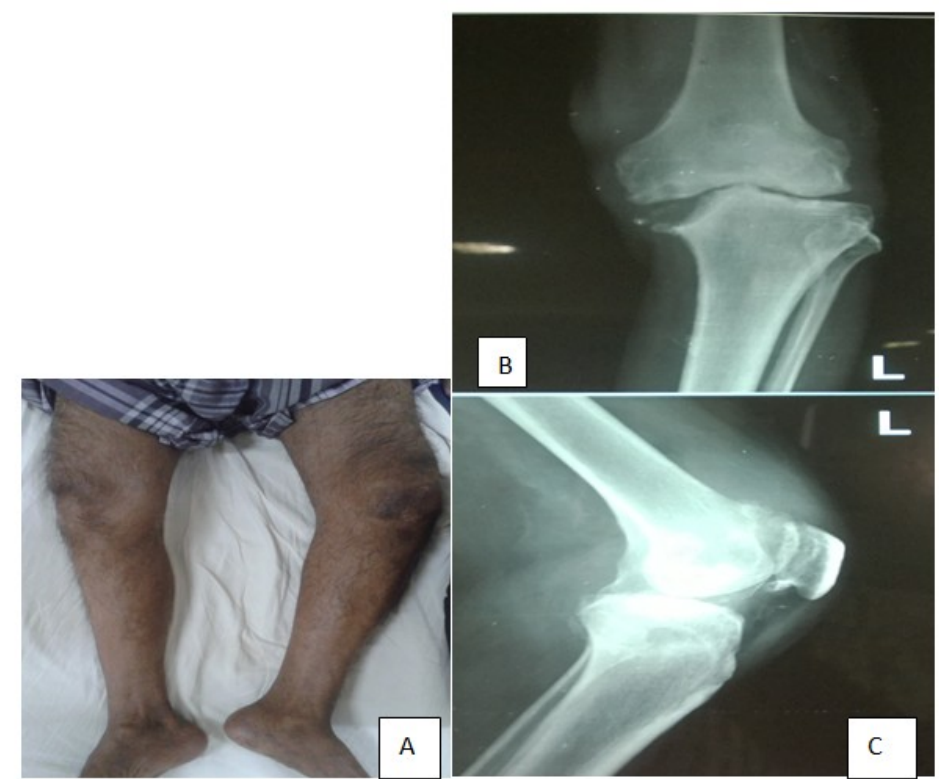

Figure 1(a,b,c): Clinical photo before surgery showing severe varus deformity of the left knee(a).Pre-opanteroposterior Xray showing medial tibial defect on left knee (b).Pre-op Lateral view of left knee 


\section{Case Presentation:}

65 year old male patient came to us with severe pain over the left knee since 10 years with difficulty in walking more than 20 feet without support.He also had difficulty in squatting,sitting crossed leg and climbing stairs.Clinically patient had medial joint line tenderness. He had varus deformity of 20 degrees and fixed flexion deformity of 15 degrees.Theflexion was noted to be 15 to 70 degrees beyond which it was painful.Standinganteroposterior and lateral radiographs of the knee were taken which showed a Type 3 of Anderson Research Institute Classification. After evaluation patient was posted for total knee replacement through routine medial parapatellarapproach.On exposure the medial tibial defect was noted to be a slope measuring $25 \mathrm{~mm}$. Routine femoral and tibia cuts were made.Tibia was cut $8 \mathrm{~mm}$ measured from the unaffected lateral plateau.The defect on the tibia was sclerosed, which was cut obliquely to freshen the edges for graft incorporation $^{2}$ (Sculco's Technique).From the femoral cuts, bone was fashioned to fit into the defect remaining after the tibialcuts.After the tibial cuts defect was measured to be $15 \mathrm{~mm}$.

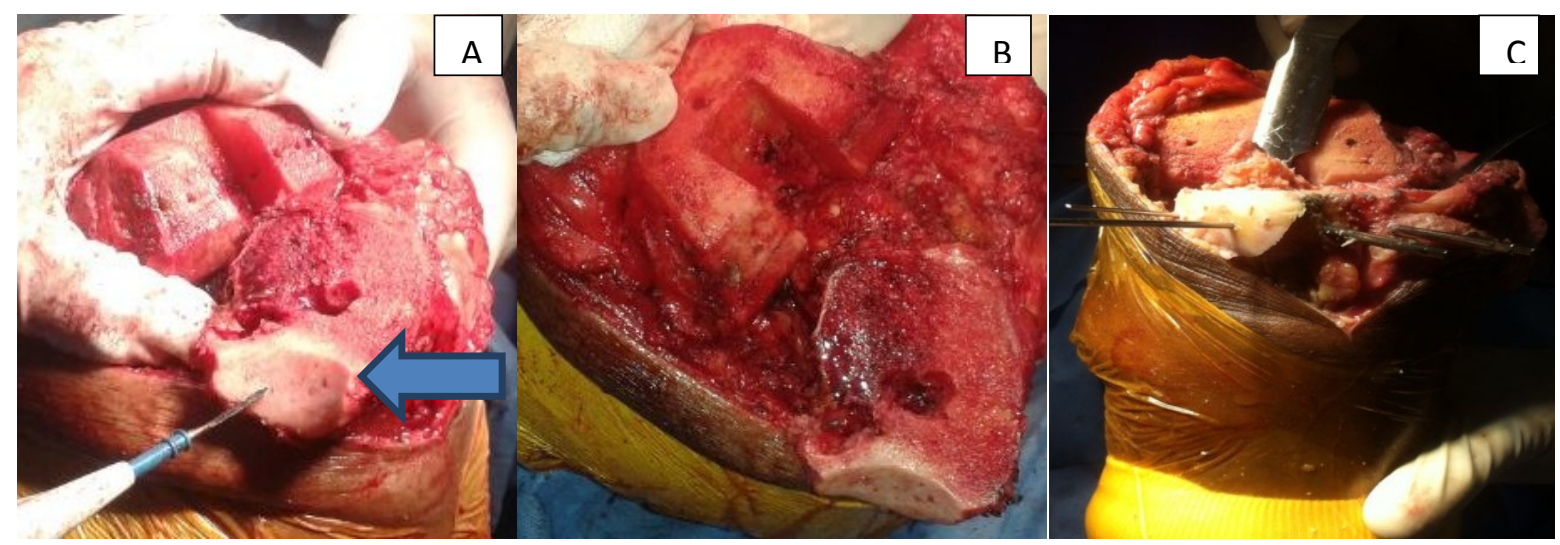

Figure2(a,b,c): Blue arrow shows the defect in the medial aspect of tibia(a).Axial view showing the medial defect(b).Graft from the femoral cuts fixed with two K wires(c).

The autograft was then fixed with two $3.5 \mathrm{~mm}$ cancellous screws with washer.Post operatively patient was started on quadriceps strengthening and knee range of movements exercises.Patient was on non weight bearing walker mobilisation for 2 months for graft incorporation.

Patient was reviewed monthly on OPD basis. After 2 months patient was advised partial weight bearing on the operated knee and then complete weight bearing walking was allowed from the third month following surgery.After 6 months follow up, patient is completely painfree, walking full weight bearing without support.Flexion is from 0 to 110 degrees.

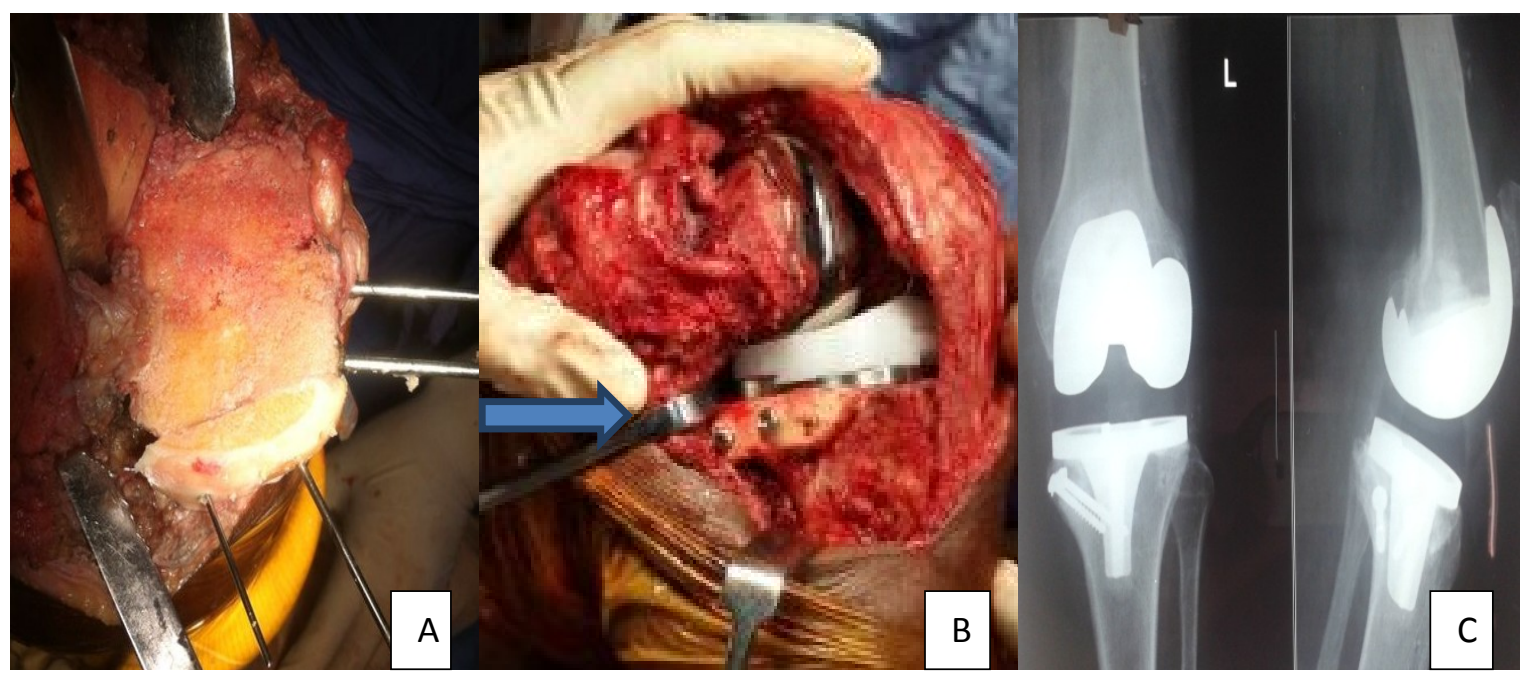

Figure 3(a,b,c): Axial view showing temporary fixation of graft with two $\mathrm{k}$ wires(a).Graft fixed with two cancellous screws after fixing the tibial plate(b).Post op xray showing the antero-posterior and lateral views 


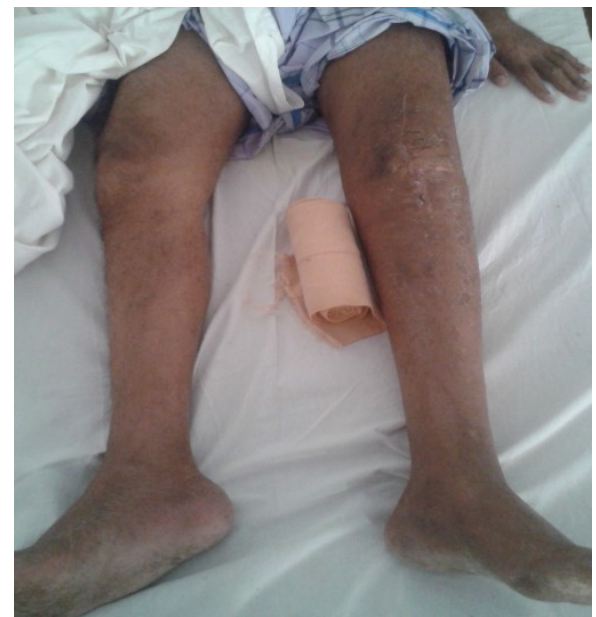

Figure 4: Post operative clinical picture showing he deformity being corrected with healed scar in the midline.

\section{Discussion:}

As mentioned earlier various methods are described for the reconstruction of the tibial plateau like Polymethacrylate cementfiller,Lowertibialresection,metallic wedge augments, autograft and custom implants.

Polymethylmethacrylate cement can be used a filler for very small defects with or without screws.These are ideal when the defect is less than $5 \mathrm{~mm}$ after completing the tibial cuts.

The limit of tibial resection is till the level of Iliotibial band ie about $10 \mathrm{~mm}$.Excessive tibial resection may lead to altered patella-femoral kinematics,poor quality cancellous bone and sizing problems.

The use of structural allograft has various disadvantages like donor availability,latereabsorption,nonunion,re-fracture and risk of disease transmission.

The use of metallic augments and custom made implants allows selective filling of the bone deficiencies in tibia. These are used when the tibial defect fails to support more than $25 \%$ of the tibial base plate. They allow immediate weight bearing and long term stability.Disadvantages of using wedges are that they are expensive,cement fatigues with time,screws produce metallic by-products and wedges doesnot restore the bone stock.

All the above mentioned limitations and disadvantages can be overcome if we use autograft.In our case the defect after tibial cuts was measuring between $15-18 \mathrm{~mm}$. Oblique osteotomy was done to remove the sclerotic bone.We could reconstruct the defect using the bone cuts taken from the distal femur.Autograft can be then stabilised temporarily with $\mathrm{k}$ wires and after final tibial base plate is fixed the graft can be stabilised with cancellous screws avoiding the tibial prosthesis ${ }^{3,4}$.The advantages of using autograft is that it is inexpensive,available from the site of surgery,helps in graft incorporation,no risk of non union,infection or disease transmission.

There was no screw loosening,graft failure or infection in our study.

\section{Conclusion:}

Sculco's technique of medial tibial defect reconstruction in primary total knee replacement using autograft is a very simple procedure which does not add to the financial burden of total knee replacement. More over it overcomes the other complications associated with other methods of reconstruction mentioned above. We believe that medial tibial defect upto $25 \mathrm{~mm}$ in the preoperative Xray can be well managed by Sculco's technique of oblique osteotomy and graft fixation.

\section{Consent:}

Written informed consent was taken from the patient for publication of the case report and accompanying images.

\section{References}

[1]. Engh G.A classification of bone defects in revision total knee arthroplasty.Presented at the Knee Soceity Interim Meeting,Philadelphia,Pennsylvania 1992

[2]. AltchekD,SculcoTP,RawlinsB.Autogenous bone grafting for severe angular deformity in total knee arthroplasty. J Arthroplasty 1989: 4(2):151-55

[3]. Dorr LD, Ranawat CS, Sculco TP, McKaskillB,Oriesek BS. Bone grafting for tibial defects in total knee arthroplasty.ClinOrthop 1986;205:153-65

[4]. WindsorRE,Insall JN, SculcoTP.Bone grafting of the tibial defects in primary and revision total knee arthroplasty. ClinOrthop 1986;205:132-7 
Figure Legend

Figure 1(a,b,c): Clinical photo before surgery showing severe varus deformity of the left knee(a).Pre-opanteroposteriorXray showing medial tibial defect on left knee (b).Pre-op Lateral view of left knee

Figure2(a,b,c): Blue arrow shows the defect in the medial aspect of tibia(a).Axial view showing the medial defect(b).Graft from the femoral cuts fixed with two K wires(c).

Figure 3(a,b,c): Axial view showing temporary fixation of graft with two $\mathrm{k}$ wires(a).Graft fixed with two cancellous screws after fixing the tibial plate(b).Post op xray showing the antero-posterior and lateral views.

Figure 4: Post operative clinical picture showing he deformity being corrected with healed scar in the midline. 